\title{
ERRATUM
}

\section{The care experiences of patients who die in residential hospice: A qualitative analysis of the last three months of life from the views of bereaved caregivers-ERRATUM}

\author{
DARYL BAINBRIDGE, MOHANNA GIRUPARAJAH, HANYAN ZOU AND HSIEN SEOW
}

doi: https://doi.org/10.1017/S147895151700058X, Published by Cambridge University Press, 29 June 2017.

In Table 4 in the original online article contained text errors under the sub headings of "Hospital care themes, Good themes" the word "homecare" should have been "hospital care". The entire corrected Table is reprinted herein.

The author and publisher regret these errors.

\section{REFERENCE}

Daryl Bainbridge, D., Giruparajah, M., Zou, H., Seow, H. (2017). The care experiences of patients who die in residential hospice: A qualitative analysis of the last three months of life from the views of bereaved caregivers. Palliative and Supportive Care. doi:10.1017/S147895151700058X. 
Table 4. Unique good and bad themes about home and hospital care

Homecare themes $(n=379)$

\begin{tabular}{|c|c|c|c|}
\hline Good themes & $n(\% *)$ & Bad themes & $n(\% *)$ \\
\hline $\begin{array}{l}\text { Generally good homecare, unspecified } \\
\text { provider }\end{array}$ & $101(26.6)$ & $\begin{array}{l}\text { Needed more staff and/or services to support patient } \\
\text { in home }\end{array}$ & $36(9.5)$ \\
\hline Generally good homecare care by nurses & $71(18.7)$ & Issues with homecare in general & $16(4.2)$ \\
\hline $\begin{array}{l}\text { Generally good homecare care by personal } \\
\text { support workers }\end{array}$ & $37(9.8)$ & $\begin{array}{l}\text { Would like the same homecare providers to come to } \\
\text { the home }\end{array}$ & $15(4.0)$ \\
\hline $\begin{array}{l}\text { Generally good homecare care by } \\
\text { occupational therapists/ } \\
\text { physiotherapists }\end{array}$ & $5(1.3)$ & $\begin{array}{l}\text { Scheduling issues with homecare services and } \\
\text { providers }\end{array}$ & $13(3.4)$ \\
\hline $\begin{array}{l}\text { Compassionate, caring, supportive, and/or } \\
\text { empathic homecare providers } \\
\text { (unspecified) }\end{array}$ & $18(4.7)$ & Getting access to homecare services was difficult & $11(2.9)$ \\
\hline $\begin{array}{l}\text { Compassionate, caring, supportive, and/or } \\
\text { empathic homecare nurses }\end{array}$ & $7(1.8)$ & $\begin{array}{l}\text { Homecare provider(s) lacked training and/or } \\
\text { experience (including questionable practices) }\end{array}$ & $10(2.6)$ \\
\hline $\begin{array}{l}\text { Compassionate, caring, supportive, and/or } \\
\text { empathic homecare personal support } \\
\text { workers }\end{array}$ & $7(1.8)$ & $\begin{array}{l}\text { Homecare needed to be better organized and } \\
\text { coordinated }\end{array}$ & $10(2.6)$ \\
\hline Support enabled patient to remain at home & $14(3.7)$ & $\begin{array}{l}\text { Care and services from unspecified homecare } \\
\text { provider lacking }\end{array}$ & $10(2.6)$ \\
\hline Responsive homecare & $9(2.4)$ & $\begin{array}{l}\text { Homecare case manager was insensitive, lacked } \\
\text { empathy, unpleasant, or lacked respect }\end{array}$ & $9(2.4)$ \\
\hline $\begin{array}{l}\text { Good pain and/or symptom management in } \\
\text { home }\end{array}$ & $9(2.4)$ & $\begin{array}{l}\text { Care and services from personal support worker(s) } \\
\text { lacking }\end{array}$ & $9(2.4)$ \\
\hline $\begin{array}{l}\text { Homecare providers took time to answer } \\
\text { questions and educate patient and family }\end{array}$ & $6(1.6)$ & $\begin{array}{l}\text { Inadequate pain and/or symptom management at } \\
\text { home }\end{array}$ & $6(1.6)$ \\
\hline $\begin{array}{l}\text { Regularity in homecare providers (i.e., the } \\
\text { same providers visit) }\end{array}$ & $6(1.6)$ & $\begin{array}{l}\text { Homecare nurse(s) insensitive, lacked empathy, } \\
\text { unpleasant, or lacked respect }\end{array}$ & $6(1.6)$ \\
\hline Respectful homecare & $6(1.6)$ & $\begin{array}{l}\text { Equipment needed at home was slow to arrive, slow } \\
\text { to be removed, did not come when scheduled, and/ } \\
\text { or did not accompany caregiver education or } \\
\text { assistance }\end{array}$ & $6(1.6)$ \\
\hline \multirow[t]{4}{*}{ Specialized equipment and care at home } & \multirow[t]{4}{*}{$6(1.6)$} & Inconsistent quality and amount of homecare & $6(1.6)$ \\
\hline & & Private homecare costs too high & $6(1.6)$ \\
\hline & & $\begin{array}{l}\text { Homecare providers' communication with family } \\
\text { and patient lacking }\end{array}$ & $5(1.3)$ \\
\hline & & Needed more doctor visits at home & $5(1.3)$ \\
\hline
\end{tabular}

Hospital care themes $(n=306)$

\begin{tabular}{|c|c|c|c|}
\hline Good themes & $n(\% *)$ & Bad themes & $n(\% *)$ \\
\hline $\begin{array}{l}\text { Generally good hospital care, unspecified } \\
\text { provider }\end{array}$ & $30(9.8)$ & Issues with hospital care in general & $56(18.3)$ \\
\hline Generally good hospital care by physicians & $16(5.2)$ & $\begin{array}{l}\text { Hospital care provider was insensitive, lacked } \\
\text { empathy, unpleasant, or lacked respect }\end{array}$ & $23(7.5)$ \\
\hline Generally good hospital care by nurses & $16(5.2)$ & $\begin{array}{l}\text { Needed more and better communication with } \\
\text { hospital care providers }\end{array}$ & $23(7.5)$ \\
\hline \multirow[t]{6}{*}{$\begin{array}{l}\text { Compassionate, caring, supportive, and/or } \\
\text { empathic hospital providers }\end{array}$} & $6(2.0)$ & $\begin{array}{l}\text { Needed more staff and/or services to support } \\
\text { patients in hospital }\end{array}$ & $17(5.6)$ \\
\hline & & $\begin{array}{l}\text { Inadequate pain and/or symptom management in } \\
\text { hospital }\end{array}$ & $13(4.2)$ \\
\hline & & More responsive hospital care needed & $13(4.2)$ \\
\hline & & $\begin{array}{l}\text { Inadequate accommodations (including bed) in } \\
\text { hospital }\end{array}$ & $9(2.9)$ \\
\hline & & $\begin{array}{l}\text { More communication and coordination needed } \\
\text { between hospital care providers }\end{array}$ & $9(2.9)$ \\
\hline & & Personal care at hospital was lacking & $5(1.6)$ \\
\hline
\end{tabular}

* The calculated percentages reflect the number of patients who received care in the setting. 